\title{
Pumps in Wearable Ultrafiltration Devices: Pumps in Wuf Devices
}

\author{
Paolo Armignacco ${ }^{\mathrm{a}}$ Francesco Garzotto $^{\mathrm{a}}$ Corrado Bellini $^{\mathrm{b}}$ Mauro Neri ${ }^{\mathrm{a}}$ \\ Anna Lorenzin $^{\mathrm{a}}$ Marco Sartori $^{\mathrm{a}}$ Claudio Ronco $^{\mathrm{a}}$ \\ ${ }^{a}$ Department of Nephrology, San Bortolo Hospital, Vicenza and ${ }^{b}$ RAND, Medolla (Modena), Italy
}

\section{Key Words}

Wearable artificial kidney · Pumping system · Blood pumps · Ideal pump

\begin{abstract}
The wearable artificial kidney (WAK) is a device that is supposed to operate like a real kidney, which permits prolonged, frequent, and continuous dialysis treatments for patients with end-stage renal disease (ESRD). Its functioning is mainly related to its pumping system, as well as to its dialysategenerating and alarm/shutoff ones. A pump is defined as a device that moves fluids by mechanical action. In such a context, blood pumps pull blood from the access side of the dialysis catheter and return the blood at the same rate of flow. The main aim of this paper is to review the current literature on blood pumps, describing the way they have been functioning thus far and how they are being engineered, giving details about the most important parameters that define their quality, thus allowing the production of a radar comparative graph, and listing ideal pumps' features.
\end{abstract}

(c) 2015 S. Karger AG, Basel
C 2015 S. Karger AG, Basel

0253-5068/15/0393-0115\$39.50/0

\section{The WAK: What It Is and the Different Conceptions of WAK}

It is well known that the prevalence of chronic kidney disease $(\mathrm{CKD})$ is progressively increasing, with all registries showing continued growth in the number of patients needing renal replacement therapy. The social and economic consequences are very important and morbidity and mortality in patients with end-stage renal disease (ESRD) remain unacceptably high.

A growing body of literature points out that increased dialysis frequency and prolonged dialysis (preferably daily) are conducive to numerous improvements in quality of life and potentially increased longevity of ESRD patients. Prolonged and frequent dialysis treatments are a much-desired goal in the treatment of ESRD, yet logistically are very difficult to accomplish [16].

A wearable artificial kidney (WAK) provides a practical and feasible solution to satisfy this need and it might resolve many issues in this regard; a few groups have been working on such a project for more than two decades. 
In particular, an ideal WAK would be wearable, thus comfortable, as well as lightweight. This would enable the patient in enjoying several advantages in performing the activities of daily living (ADLs), such as walking and working, or even cooking, jogging, and other similar activities. It would even be highly efficient since it is expected to operate like the real kidney, primarily allowing the necessary filtration rate. It should moreover allow the maintenance of the acid-base balance and of the hydroelectrolytic equilibrium.

Till now, the WAK has already been conceptualized in several ways:

- ViWAK (Vicenza WAK) [1,21-23]

- BioRAD (Bioartificial Renal Assist Device) [3]

- HNF (Human Nephron Filter) [4]

- CARPEDIEM (Cardio-Renal Pediatric Dialysis Emergency Machine) $[5,21]$

- AWAK (Automated WAK) [22, 23]

- WAKMAN [5, 21-23].

\section{Gura Current and Ideal WAK}

In order to achieve a true wearability, the WAK had to encounter and solve some challenges, such as those related to its safety, ergonomics, lightweight but powerful battery design, prevention of clotting, and toxicity rates limitation.

A somewhat detailed representation of the current WAK system, engineered by V. Gura, and the prototype WAKMAN designed in Vicenza is given in figure 1.

Its working principle is based on drawing blood from a double lumen catheter (red), anticoagulating it with heparin from a reservoir (white) using a micro pump and then circulating it through the blood channel of the WAK shuttle pump (gray) and into the dialyzer. The blood returns then to the venous side of the double lumen catheter (blue), and clean dialysate (green) enters the dialyzer. The dialysate circulates in countercurrent flow to the blood and exits (yellow) into the dialysate channel of the WAK pump. Another pump removes a predetermined amount of the spent dialysate (yellow) into a collection bag. The dialysate then returns to the dialyzer (green) [16], after going through a series of sorbents and being infused with a solution containing sodium bicarbonate.

The flexible design would be positioned on the user to mimic a functioning kidney, and it would be discrete and comfortable, so to allow patients to move freely with little thought of the device.

The requirements for new technologies in dialysis, in effect, are based on the following objectives:
- Safety;

- Biocompatibility;

- Ease of use;

- Dependability;

- Continuous power supply;

- Portability, or, even better, Implantability;

- Limited mechanical noise;

- Elimination of molecular weight solutes similar to kidney function;

- Elimination of water and solutes according to the patient's need.

The weight, shape, and form of the WAK have to allow ergonomic adaptation to the body contour so that it can be worn continuously and permit the patient to sleep, sit, and walk comfortably.

Above all, it should be easy to use and dependable, so that it can be used without the frequent need of expert assistance, enabling reliability, maintainability, availability, and security.

Moreover, freedom from the electrical outlet is required even though there is a need for enough energy to propel blood and dialysate through the device. This means that either batteries or pumps are needed - batteries should be light but still deliver enough power and pumps should be highly efficient but consume less power.

In such a context, this paper is intended to analyze the WAK pumping system more in detail.

\section{The Wearable Artificial Kidney and Its Pumping System}

A pump can be generically defined as a device that moves fluids (liquids or gases) by mechanical action.

It is an apparatus for drawing or forcing liquids or gases; that is, it is a mechanism that mediates active transport of ions or molecules across a biological membrane; thus, a pump is a device that is used to propel blood through the tubing of extracorporeal circulation tools.

In standard renal therapies, small lightweight minipumps are required to reduce the complexity load of the circuit due to clotting prevention and to ultrafiltration and anticoagulation volumes monitoring. The traditional hemodialysis circuit encompasses a venous blood chamber, which acts as a safety device to prevent air emboli returning to the patient. As blood-air interfaces increase the risk of clot formation in the extracorporeal circuit, this standard design has to be changed for wearable devices, by utilizing mini-pumps in gas-permeable or water-impermeable plastics to remove micro-bubbles. 


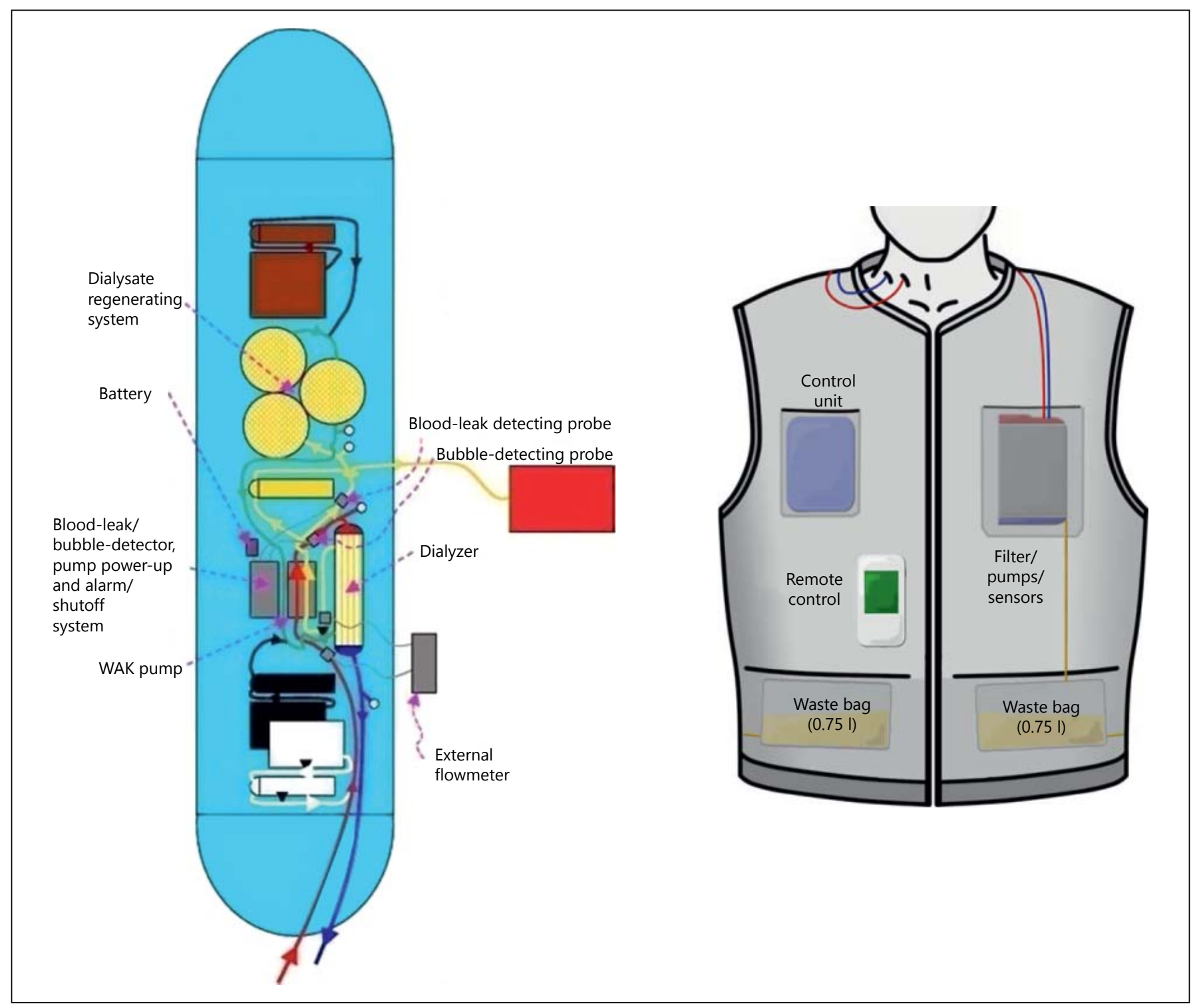

Fig. 1. Schematics of the current Gura WAK system and the prototype Vicenza WAKMAN.

Pumps used in the WAK belong to the group of dialysis pumps. It is possible to distinguish several kinds of dialysis pumps. The two most important typologies are blood and infusion pumps, which are both described in this paper, although special focus is on blood pumps, given their importance in the WAK working principles.

In the WAK, to be truly portable, an energy-efficient pump, capable of being powered by long-life lightweight batteries without generating excess heat, is required. As such, several different pump designs have been suggested especially to provide adequate flows.

Pumps in Wearable Ultrafiltration Devices

\section{Blood Pumps}

Blood pumps pull blood from the access side of the dialysis catheter and return the blood at the same rate of flow [19].

The use of blood pumps provides a reliable and adjustable blood flow through the filter. The constant blood flow provided by the pump decreases the risk that the filter will clot.

\section{Infusion Pumps}

An infusion pump is a medical device used to infuse fluids into a patient's body in a controlled manner. 
Infusion pumps may be capable of delivering fluids in large or small amounts, and may be used to deliver nutrients, medications, anticoagulants, and antibiotics.

They can be designed for stationary use at a patient's bedside, or can be made to be portable or wearable (ambulatory infusion pumps).

They can be large-volume or small-volume pumps while the former infuse nutrient solutions, the latter infuse hormones.

The most important types of infusion pumps are:

- Replacement pumps: They deliver IV therapy (IntraVenous therapy) at a prescribed rate, allowing a fluid deliver into the blood circuit whether before or after the filter;

- Effluent pumps: They pull plasma water from the patient's blood stream across the dialysis filter at a preset volume;

- Pre-blood pumps (PBPs): They deliver the replacement fluid into the blood circuit immediately after the blood is pulled into the circuit at the access site, so they can be used to deliver anticoagulants, ensuring they enter the blood circuit as soon as possible [15];

- Passive/active-drug-infusion pumps: Passive-infusion and active-infusion pumps respectively infuse by means of an external force, or by an actively produced transport. Passive-type drug infusion pumps have several advantages over the active-type ones, like their simple drug chamber structure and their relatively high operational stability. However, they also have several limitations, like a fixed flow-rate and monotonic flow patterns [18].

\section{Parameters Defining a Pump's Quality}

The quality of a pump can be determined based on several parameters. The main ones are the pump's safety, biocompatibility, MTBF (mean time between failures), flow rate, hemolysis, and cost; they can all be established once the pump's size, weight and energy consumption are fixed.

Safety is the state of being 'safe', the condition of being protected against any consequence of failure, damage, error, accident, harm, or any other event that could be considered non-desirable.

Biocompatibility is related to the behavior of biomaterials in various contexts. The term refers to the ability of a material to perform with an appropriate host response in a specific situation. Modern medical devices are often made of more than one material so it might not always be sufficient to talk about the biocompatibility of a specific material.
Mean time between failures (MTBF) is the predicted time that elapses between inherent failures of a system during operation. MTBF can be calculated as the arithmetic mean (average) time between failures of a system. For complex, repairable systems, failures are considered to be those events that occur due to design conditions, which place the system out of service and move it into a state for repair.

The volumetric flow rate is the volume of fluid that passes through a given surface per time unit. Its SI unit of measurement is $\left[\mathrm{m}^{3} / \mathrm{s}\right]$. The flow rate through a surface $S$ is calculated as:

$$
\int \mathrm{J}_{\mathrm{f}} \cdot \mathrm{n} \mathrm{dS},
$$

where $\mathrm{J}_{\mathrm{f}}$ is the physical quantity flux and $\mathrm{n}$ is perpendicular to the surface versor.

Flux, relating it with transport phenomena (heat transfer, mass transfer, and fluid dynamics), is defined as the rate of flow of a property per unit of area; its dimensions are [quantity].[time] ${ }^{-1} \cdot[\text { area }]^{-1}$.

Hemolysis is the rupturing of erythrocytes and the release of their contents (cytoplasm, hemoglobin) into the surrounding fluid (e.g., blood, plasma).

Cost is the amount that has to be paid or given up in order to get something.

It is also possible to introduce several other parameters that are useful when describing a pump's quality.

First of all, in the fields of science, engineering, industry, and statistics, the accuracy [10] of a measurement system is the degree of closeness of measurements of a quantity to that quantity's actual (true) value. The precision [10] of a measurement system, also called reproducibility or repeatability, is the degree to which repeated measurements under unchanged conditions show the same results [11].

The weight of an object $[12,13]$, is taken to be the force on the object due to gravity. Its magnitude, often denoted with $\mathrm{W}$, is the product of the mass $m$ of the object and the magnitude of the local gravitational acceleration $g$ [14]; thus: $\mathrm{W}=\mathrm{mg}$; its unit of measurement is that of force, which in the SI system is, Newton $[\mathrm{N}]$.

About energy consumption, it is the amount of energy or power consumed in a process or system.

Ergonomics indeed is the scientific discipline concerned with the understanding of interactions among humans and other elements of a system, and the profession that applies theory, principles, data, and methods to design in order to optimize human well-being and overall system performances. 
Resistance is a mechanism that creates dissipation. Mechanisms causing this dissipation are varied and depend on many factors; thus, it is possible to distinguish several kinds of resistance, such as thermal, usury, static, and dynamic resistance.

The number of moving parts is a factor that affects vibration or the manufacturing cost or durability. Consequently, a low number of moving parts yields a better pump for the portable system.

The contact area between blood and moving parts and the duration time would better be as low as possible, since they are hemolysis-inducing factors.

Infections (deriving from the contact between the blood inside and outside the pump) should be completely avoided, so there does not have to be any connection between the blood inside and outside the pump.

The temperature should be kept $\leq 37.5^{\circ} \mathrm{C}$ and the friction at the contact point would be low.

It is important that during operation there should not be distracting noises, and pumps' maintenance would have minimal blood clogging (thanks to the use of valves) and would allow patients to visit the clinic less frequently, thus making it easy to keep the pump clean at home.

\section{Types of Pumps}

\section{Positive Displacement Pumps}

A positive displacement (PD) pump is a pump that creates a rise in pressure by cyclic filling and emptying of a volume.

A PD blood pump makes a fluid move by trapping a fixed amount and forcing (displacing) that trapped volume into the discharge pipe.

Positive displacement pumps operate with a series of working cycles. Each cycle encloses a certain volume of fluid and moves it mechanically through the pump into the system. Depending on the type of pump and the liquid being handled, this happens with little influence from the back pressure on the pump.

Some PD pumps use an expanding cavity on the suction side and a decreasing cavity on the discharge side. Liquid flows into the pump as the cavity on the suction side expands, and the liquid flows out of the discharge as the cavity collapses. PD pumps have a component that separates the fluid from the energy-imparting component. Allowing direct contact with the fluid and the pump, actuation parts could lead to possible contamination and blood clotting. PD pumps, unlike centrifugal pumps, produce the same flow at a given speed, no matter the discharge pressure.

Pumps in Wearable Ultrafiltration Devices
This type of pump moves blood forward by displacing the liquid progressively, from the suction to the discharge.

A major advantage of a positive displacement pump is its ability to deliver consistent capacities because the output is solely dependent on the basic design of the pump and the speed of its driving mechanism. This means that if the liquid is not moving through the system at the required flow rate, it can always be corrected by changing one or both of these factors.

Positive displacement pumps can be further classified according to the mechanism used to move the fluid:

- Rotary-type positive displacement: they consist of a cylindrically bored housing with a suction inlet on one side and a discharge outlet on the other;

- Reciprocating-type positive displacement: piston or diaphragm pumps;

- Linear-type positive displacement: rope pumps and chain pumps.

\section{Piston Pumps}

The oldest and best known PD pump is the piston pump. It uses a piston or plunger to force liquid from the inlet side to the outlet side of the pump.

As the piston moves upwards, it will reduce the pressure in the pump body. This causes the pressure in the suction line to open the suction valve and permit the liquid to flow into the pump. In the same way, the higher pressure in the discharge line keeps the discharge valve closed. This is called the 'suction cycle'. When the plunger moves downwards, it increases the pressure in the body, closing the suction valve and opening the discharge valve to force the liquid out of the pump. This is called the 'discharge cycle'. The movement of the plunger inside the pump body creates pressure inside the pump. Because of this, you must ensure that a piston pump is never operated against a closed discharge valve. All discharge valves must be open before the pump is started to prevent a quick increase of pressure that could damage the pump or the system (table 1).

\section{Peristaltic Pumps}

A peristaltic pump is a type of positive displacement pump that is used during hemodialysis.

In general, any pump used in a biological system must not introduce harmful agents (such as bacteria or viruses) to the blood or dialysate to maintain sterility. In such a context, peristaltic pumps can easily provide this safeguard and adequately perform with the flow characteristics of the system. 
Table 1. The values of the most important parameters

\begin{tabular}{|c|c|c|c|c|c|c|c|c|}
\hline PD pumps & 2.5 & 4.5 & 2 & 2.5 & 2 & 4 & 2 & 2 \\
\hline Peristaltic pumps & 0.5 & 3 & 3 & 1.5 & 2.5 & 3.5 & 2.5 & 1 \\
\hline Dynamic pumps & 2.5 & 4.5 & 3 & 3 & 3.5 & 3 & 2.5 & 3.5 \\
\hline \multicolumn{9}{|l|}{ Kinetic centrifugal } \\
\hline Shuttle pumps & 1.5 & 4 & 1.5 & 2.5 & 3 & 4 & 3 & 2 \\
\hline Rotary pumps & 2.5 & 4 & 4 & 3.5 & 2 & 3.5 & 3.5 & 0.5 \\
\hline
\end{tabular}

The values are expressed in arbitrary units in a range $(0 ; 4.5)$, where the higher the number is, the better the evaluated pump's parameter is.

What makes a peristaltic pump function smoothly and efficiently are the brushless DC motors that are situated in the pump head.

Brushless DC motors are more reliable and have a higher life expectancy than brushed DC motors; this difference is largely due to the fact that brushless DC motors do not have brushes that need to be replaced at regular intervals. Brushless DC motors help to transfer heat away from the motor, a feature that helps the motor to run cool in a crowded place. They provide greater amounts of torque over a larger speed range and higher duty cycles than brushed DC motors. Moreover, when situated in peristaltic pumps, brushless DC motors are quieter, have less electromagnetic interference, and do not produce sparks from friction against a commutator.

Peristaltic blood pumps use flexible tubing to run through rollers in the pump head. As the fluid goes through the pump head, packets of fluids are formed. The flow rate is determined by the size of the packets and the speed of the rollers turning.

Moving rollers in a peristaltic pump squeeze a flexible tube forcing the fluid inside to move in one direction. Because the flexible tubing is the only part exposed to the fluid, the pump can easily maintain its sterility.

The ideal peristaltic pump should have an infinite diameter of the pump head and the largest possible diameter of the rollers. Such an ideal peristaltic pump would offer an unlimited tubing lifetime and provide a constant and pulsation-free flow rate. Ideal peristaltic pumps cannot be constructed in reality; however, they can be designed to approach these ideal parameters [20].

With regard to their lifetime, pumps have an adequate lifetime because only the flexible tubing requires replace- ment at regular intervals, and they are noted for their superior metering abilities.

This sort of pumps has many advantages; the two most important ones are:

- The only part of a peristaltic pump that comes in contact with the liquid is the tubing. This maintains the sterility of the fluid and of the pump;

- A peristaltic pump is non-siphoning, which means that it prevents back the flow into the system. This promotes accuracy during dispensing.

In relation to the WAK, Gura et al. have already developed a specialized peristaltic pump for their prototype, which has not been commercialized yet (table 1).

\section{Dynamic Pumps}

Dynamic pumps are one category of pumps under which there are several classes, like centrifugal and axial ones.

These pumps operate by developing a high liquid velocity and converting the velocity to pressure in a diffusing flow passage. Dynamic pumps usually have lower efficiencies than positive displacement pumps, but also have lower maintenance requirements. Dynamic pumps are also able to operate at fairly high speeds and high fluid flow rates (table 1).

\section{Dynamic Centrifugal Pumps}

A centrifugal pump consists of an impeller and an intake at its center. These are arranged so that when the impeller rotates, liquid is discharged by centrifugal force into a casing surrounding the impeller. The casing is there in order to gradually decrease the velocity of the fluid that leaves the impeller at a high velocity. This velocity is converted to pressure, which is needed to discharge the fluid. Some of the advantages of centrifugal pumps are smooth 
flow through the pump and uniform pressure in the discharge pipe, low cost, and an operating speed that allows for direct connection to steam turbines and electric motors. The centrifugal pump accounts for not less than $80 \%$ of the worlds' pump production since it is more suitable for handling large capacities of liquids than positive-displacement pumps (table 1).

\section{Axial Flow Pumps}

Axial flow pumps are also called Propeller pumps. These pumps develop most of their pressure by the propelling or lifting action of the vanes on the liquid. They are often used in wet-pit drainage, low-pressure irrigation, and storm-water applications.

In general, vertical single-stage axial and mixed-flow pumps are used, but sometimes two-stage axial-flow pumps are economically more practical. Horizontal axialflow pumps are used for pumping large volumes against low pressures and usually employ a siphonic action.

When higher pressures are involved, these pumps can be arranged to operate with siphonic action until the back pressure places the hydraulic gradient above the pump.

\section{Kinetic Centrifugal Pumps}

A centrifugal pump is a pump that relies on an impeller inside the fluid to provide a pressure rise.

They convert the input power to kinetic energy in the liquid by accelerating the liquid with a revolving device.

The most common type is the volute pump. Fluid enters the pump through the eye of the impeller, and it is accelerated outward from the pump chasing, creating a vacuum at the impeller's eye.

The energy created by the pump is kinetic energy according to the Bernoulli Equation. The energy transferred to the liquid corresponds to the velocity at the edge of the impeller, and it is directly proportional to the revolving impeller speed and size.

Kinetic pumps have some characteristics:

- They are designed with impellers arranged with vanes or cones;

- Centrifugal pumps are magnetically driven and produce a pressure differential as they rotate.

Some advantages these pumps have are as follows:

- Reduced hemolysis;

- No dangerous inflow/outflow pressures;

- No need to calibrate.

On the other hand, the main disadvantages are as follows:

- They cause over-heating;

- Over-heating promotes clotting (table 1).

Pumps in Wearable Ultrafiltration

Devices

\section{Turbine Pumps}

Turbine pumps move a fluid along an annular path within a casing, from an inlet to an outlet. The direction of pumping may be reversed in a simple way by reversing the direction of rotation of the impeller. In many applications of reversible turbine pumps, it is desirable for the pump to have a higher flow rate in one direction than in the other; however, the usual turbine pump essentially delivers the same flow rate in both directions of rotation [21].

The turbine has an impeller positioned on a shaft and the discharged fluid engages the impeller causing the impeller and the shaft to rotate. It is positioned adjacent to the turbine to receive the fluid pumped to the process (table 1).

\section{Shuttle Pumps}

A shuttle pump employs a unique pumping principle - a combination of Rotary and Piston Pump - through two double acting pistons in their respective cylinders.

They have three pumping elements: the rotor, the piston, and the shuttle. The rotor is keyed to a single shaft and runs concentric inside the pump casing. It has a rectangular slot across its face. The channel-shaped piston reciprocates within this slot. A rectangular block, the shuttle, fits liquid tight in the piston slot and reciprocates.

Like a true piston pump, this pump can handle highly viscous liquids or thin volatile materials with unmatched suction characteristics. This pumping principle, unlike centrifugal pumps, handles the product without breaking it down, emulsifying, foaming, chewing, or heating (table 1).

The WAK shuttle pump, whose function is to provide simultaneous pulsatile flows of both blood and dialysate at a half-cycle phase difference, uses a motor, which causes an intermittent mechanism with two metal arms to alternatively compress two chambers. At the entry and exit of these chambers, valves open and close to allow an alternating pulsatile flow of both blood and dialysate into the dialyzer (fig. 2).

\section{Rotary Pumps}

Rotary pumps are the most commonly used pumps in hemodialysis. They are positive-displacement pumps that consist of vanes mounted to a rotor that rotates inside a cavity. They can include hydrodynamic, magnetic and hybrid, hydrodynamic/magnetic bearings and combinations thereof. The rotor can include a shaft, or the pump can be made shaftless. 
Fig. 2. Flow behavior of both blood $\left(^{*}\right)$ and dialysate $\left(^{*}\right)$ in the wearable artificial kidney pump. $\mathrm{Qb}=$ Blood flow rate; $\mathrm{Qd}=\mathrm{di}$ alysate flow rate. (Adapted from Gura V, Beizai M, Ezon C, Polaschegg HD: Pulsatile blood and dialysate counter phase flows, increased sorbent capacity and a high flux membrane explain the high efficiency of the Wearable Artificial Kidney. J Am Soc Nephrol 2005;16:38A-39A.)

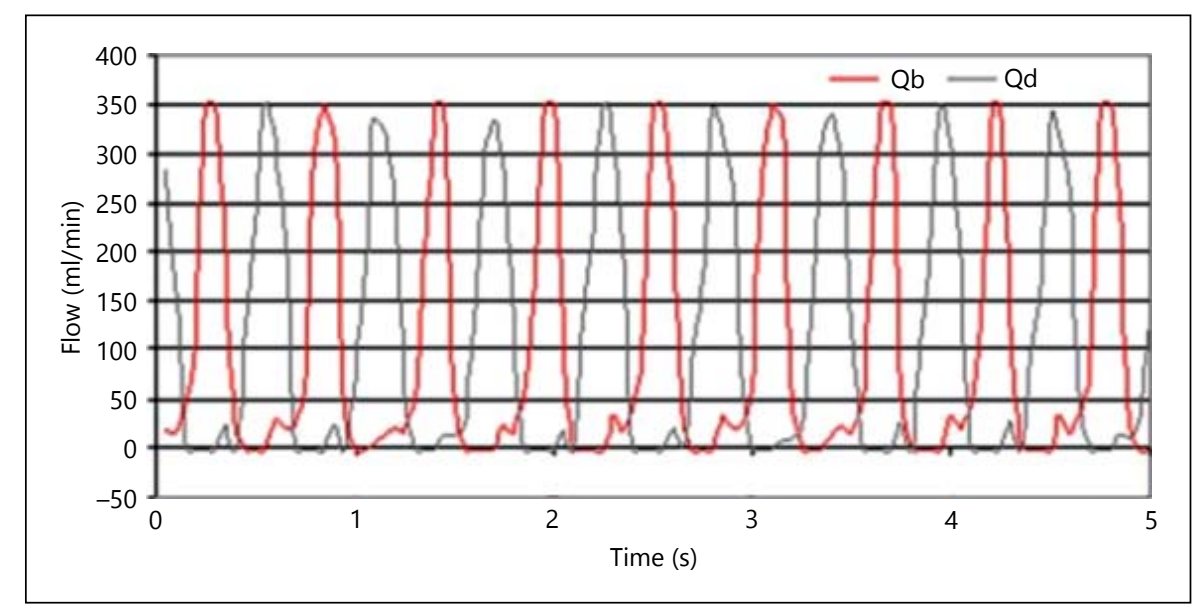

One of the principle advantages these pumps have is the gentle handling of the corpuscular blood components. This has been proven in terms of reduced hemolysis during long-term treatments [26, 27].

Another demonstrated benefit of rotary blood pumps is that they cause reduced inflammation and reduced postoperative bleeding (table 1).

\section{Rotary Gear Pump}

The external rotary gear pump is a positive displacement pump where the unmeshing of the gears produces a partial vacuum to draw the liquid into the pump. The liquid is carried between the gear teeth and the casing to the opposite side of the pump. The meshing of the gears forces the liquid into the outlet line.

The direction of pump rotation determines which of the nozzles will be the inlet and the outlet. By reversing this rotation, the function of the nozzles will be reversed and the pump will be able to pump 'backwards'.

Where one gear is driven by the other, the driven gear usually runs in sleeve type bearings. The bearings and shaft journal are located in the pump casing and surrounded by the pumped fluid. Consequently, these bearings and gears are dependent on the lubricating qualities of the pumped fluid.

Other types are available where the gears have no metallic contact with each other. In such cases, both rotors are driven by synchronized gears separated from the pump chamber. As both shafts pass through the pump casing, two sets of seals are required. The absence of metallic contact between the surfaces of the rotors and the casing means that it is insignificant to wear on these parts. The only wear that occurs is due to friction with the pumped fluid.

\section{Radar Graph and Discussion}

The following radar graph (fig. 3) compares blood pumps' performances, according to their eight most important properties.

In such a graph, eight parameters are compared for different kinds of pumps: positive displacement (blue), peristaltic (red), dynamic (green), kinetic centrifugal (violet), turbine (light blue), shuttle (orange), and rotary (grey) pumps.

The eight considered parameters, (1-8), respectively are:

1. Hemolysis

2. Biocompatibility

3. Precision, accuracy, and volumetric flow rate

4. Energy consumption

5. Weight

6. Ergonomics

7. Dimensions, size

8. Cost

The possible values these parameters can get are ranged in Arbitrary Units between 0 and 4.5, with 0 being the worst value for the considered pump, and 4.5 the best one.

From this graph, it comes into light whether that peristaltic blood pumps are the most hemolytic and energyconsuming ones, or that shuttle pumps are very ergonomic, together with positive displacement pumps, which are very biocompatible too.

Then, kinetic centrifugal, turbine, and rotary blood pumps are the most precise and accurate, and kinetic centrifugal pumps are the heaviest ones.

About their costs, dynamic and positive displacement pumps usually are the cheapest ones, while rotary pumps are the most expensive. 
Fig. 3. Radar comparative graph of seven typologies of blood pumps: positive displacement (blue), peristaltic (red), dynamic (green), kinetic centrifugal (violet), turbine (light blue), shuttle (orange), and rotary (grey) pumps.

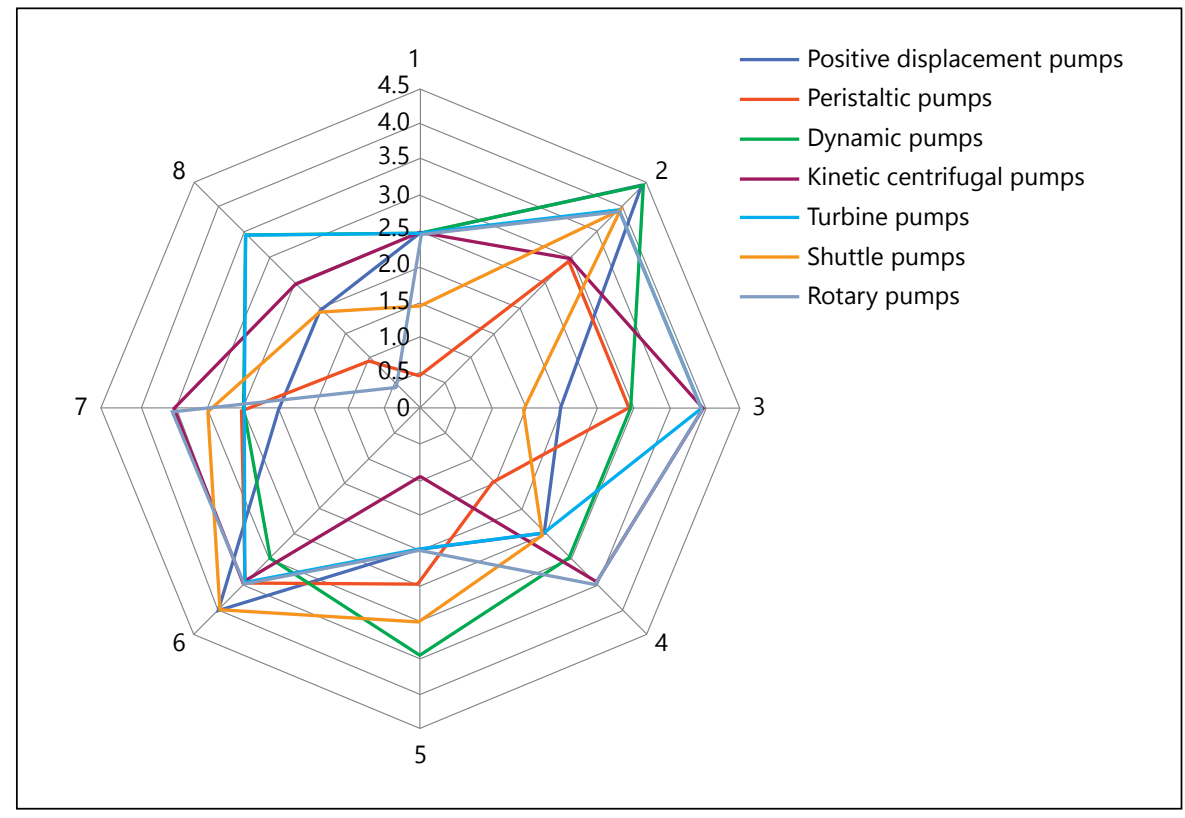

What is more, centrifugal and rotary blood pumps are the ones that fill in a smaller space.

So, to summarize, it is clear that at the moment there is not any type of dialysis WAK pump, which is entirely better than the others. As on date we do not have a WAK pump that has all the best characteristics in every considered feature integrated into it.

\section{Conclusion: The 'Perfect' Pump}

In the light of these pumps' performances comparison, the characteristics of a 'perfect pump' are listed below:

- High safety rates;

- Elevated biocompatibility (2) characteristics;

- Tending to infinite MTBF;

- Mean value and smooth-shaped flux speeds;

- Fluxes compatible with the subject's well-being;
- Low hemolysis (1);

- Dimensions (7) and costs of production (8) as low as possible.

What is more, the pump's accuracy and its precision (3) should tend to infinite values, while its energy consumption (4) and weight (5) should bottom out. Ergonomics (6) should be notable, and its resistances (thermal, usury, static, dynamic) should have intermediate values, so not to be neither too high (because the system would be too invariable) nor too low (because every little change of any parameter would cause a considerable transformation in the system performances).

\section{Disclosure Statement}

The author(s) declare(s) that there is no conflict of interest.

\section{References}

1 Ronco C, Fecondini L: The Vicenza wearable artificial kidney for peritoneal dialysis (ViWAK PD). Blood Purif 2007;25:383-388.

2 Davenport A: Portable or wearable peritoneal devices - the next step forward for peritoneal dialysis? Adv Perit Dial 2012;28:97-101.

-3 Tumlin J, Wali R, Williams W, Murray P, Tolwani AJ, Vinnikova AK, et al: Efficacy and safety of renal tubule cell therapy for acute re- nal failure. J Am Soc Nephrol 2008;19:10341040.

4 Viktorsdottir O, Palsson R, Andresdottir MB, et al: Prevalence of chronic kidney disease based on estimated glomerular filtration rate and proteinuria in icelandic adults. Nephrol Dial Transplant 2005;20:1799-1807.

5 Herrero Calvo JA: Servicio de Nefrologia. Nefrologia 2011;31:9-16.
6 AWAK Technologies Inc., 2013.

7 Fissell WH, Roy S, Davenport A: Continuous wearable hemodialysis devices. Kidney Int 2013;84:256-264.

8 Gura V, Davenport A, Beizai M, et al: Beta2-microglobulin and phosphate clearances using a wearable artificial kidney: a pilot study. Am J Kidney Dis 2009;54:104111. 
9 Gura V, Ronco C, Davenport A: The wearable artificial kidney, why and how: from holy grail to reality. Semin Dial 2009;22:13-17.

10 International Vocabulary of Metrology - Basic and General Concepts and Associated Terms (VIM 3rd edition). JCM 200:2012.

11 Taylor JR: An Introduction to Error Analysis: The Study of Uncertainties in Physical Measurements. University Science Books 2011, pp 128-129. ISBN 0-935702-75-X.

12 Morrison RC: Weight and gravity - the need for consistent definitions. The Physics Teacher 1999;37:51.

13 Galili I: Weight versus gravitational force: historical and educational perspectives. Int J Sci Educ 1999;23:1073-1093.

14 The National Standard of Canada, CAN/ CSA-Z234.1-89 Canadian Metric Practice Guide, 1989.

15 Kellum JA, Bellomo R, Ronco C: Continuous renal replacement therapy. 2009;24:129-140.
16 Gura V, Ronco C: Critical Care Nephrology, Continuous Renal Replacement Therapies, 2009, chapter 260, section 18.

17 Principles of Continuous Renal Replacement Therapy, 2005 Orlando Regional Healthcare, Education \& Development.

18 Choi SW, Kang SM, Kim HY, Nam KW: Development and in vitro evaluation of a flowadjustable elastic drug infusion pump. Proc Inst Mech Eng H 2011;225:1070-1077.

19 Veres JP: Blood Pump, Kidney Replacement Services, P.C., BiBTeX, EndNote, RefMan, US6439845 B1.

20 NMB Technologies Corporation, A Minebea Group Company, 2010.

21 Toma DN: Gen Electric, BiBTeX, EndNote, RefMan.
22 Ronco C, Jeong Chul K: The $\mu 3 \mathrm{D}$ Project (Miniaturization of three devices in dialysis), 2011.

23 Kim JC, Garzotto F, Nalesso F, et al: A wearable artificial kidney: technical requirements and potential solutions. Expert Rev Med Devices 2011;8:567-579.

24 Kim JC, Ronco C: Personal daily dialysis: the evolution of the artificial kidney. Blood Purif 2013;36:47-51.

25 Bennett M, et al: Pump-induced haemolysis: a comparison of short-term ventricular assist devices. Perfusion 2004;19:107-111.

26 Tiedge S, et al: Erste Einsätze einer neuen Diagonalpumpe in extrakorporalen Unterstützungssystemen bei Kindern und Säuglingen. Kardiotechnik 2011;3:72-76.

27 Kang J, Scholz T, Weaver JD, Ku DN, Rosen DW: Pump design for a portable renal replacement system. J Med Devices 2011;5:331340 